\title{
Necrotizing Enterocolitis: Research Agenda for a Disease of Unknown Etiology and Pathogenesis ${ }^{1}$
}

\author{
R. M. KLIEGMAN, W. A. WALKER, AND R. H. YOLKEN \\ Department of Pediatrics, Medical College of Wisconsin, Milwaukee, Wisconsin 53226 [R.M.K.]; Harvard \\ Medical School, Cambridge, Massachusetts 02138 [W.A.W.]; and Johns Hopkins University. \\ Baltimore, MD 21218 [R.H.Y.]
}

INTRODUCTION. Necrotizing enterocolitis (NEC) is a significant neonatal public health problem that affects low-birth weight infants in neonatal intensive care units throughout the country. As the survival rate of low-birth weight infants continues to increase and as the number of low-birth weight births remains unchanged, we can anticipate that NEC will continue to be a cause of significant morbidity and mortality in the future.

Despite many reports about NEC that describe demographic risk factors and short-term or long-term outcome, there is a paucity of basic science information about neonatal gastrointestinal physiology and pathophysiology in human preterm and even full-term infants. It has become increasingly evident that we need a much better understanding about the developmental aspects of gastrointestinal function in health and disease before we can achieve further advances in our understanding of and thus rational therapy for and prevention of NEC.

The purpose of the National Institute of Child Health and Human Development conference "Necrotizing Enterocolitis: Basic Science Approaches to Gut Maturation and Pathogenesis" was to bring together basic science investigators, clinical epidemiologists, and clinical scientists to identify important areas of research that need to be applied to the problem of NEC. The concept of applying the "bench to bedside" type of collaborative research was emphasized and encouraged because many clinical neonatologists may have little scientific interaction with basic scientists. In addition, many basic scientists may be unaware of NEC and the implications for targeted research related to this disease. Finally, there have been major advances in our ability to study diseases of unknown etiology, as witnessed by recent discoveries of the microbiologic agents that cause AIDS, non Anon $B$ hepatitis, roseola, and Whipple disease, and various agents that cause infectious diarrhea.

Invited participants represented a broad spectrum of basic science and clinical disciplines and included investigators with expertise in adult gastroenterology, animal physiology, cell biology, molecular virology, host defense, inflammatory mediators, vascular physiology, surgery, pathology, and neonatology. The goals of the participants were to address the gaps in our knowledge about NEC, to reconcile basic science observations about gastrointestinal disease with those in the clinical disease, and to recommend promising areas of investigation worthy of further study. This report summarizes the current concepts of neonatal gastrointestinal physiology and pathophysiology that were presented by experts in the areas relevant to NEC. The report then presents recommendations for future areas of collaborative research initiatives to fill the gaps in our knowledge about NEC. This research agenda is also the basis of a request for applications

Correspondence and reprint requests: Robert M. Kliegman, M.D., Professor and Chair, Department of Pediatrics, Medical College of Wisconsin, MACC Fund Research Center, 8701 Watertown Plank Rd., Milwaukee, WI 53226.

'Summary report of the National Institute of Child Health and Human Development conference "Necrotizing Enterocolitis: Basic Science Approaches to Gut Maturation and Pathogenesis," Bethesda, MD, October 19, 1992. from the National Institute of Child Health and Human Development to address the next stage of investigations. The names of the participants are included in the acknowledgement.

Necrotizing enterocolitis (NEC) is the most common, lifethreatening acquired gastrointestinal disease that predominantly affects premature infants in neonatal intensive care or step-down units (1). Despite hundreds of published articles on NEC since the landmark publications from Babies Hospital in New York of Mizrahi and colleagues in 1965 and Santulli and associates in 1975, NEC remains a disease of unknown etiology and uncertain pathogenesis. Treatment has been based on empiric observation and until recently prevention has not been uniformly successful.

NEC had traditionally been thought to be caused by a combination of gastrointestinal ischemia (dive reflex pattern of blood flow), gastrointestinal feeding practices and various undetermined contributions of the intestinal bacterial flora (1). Various risk factors were thought to predispose the infant to NEC (e.g. asphyxia, hypotension, respiratory distress syndrome (RDS), umbilical arterial catheterization, hypothermia, and patent ductus arteriosus). Today, after multiple case-control studies have disproved the role of these variables, we have a better understanding about predisposing factors and now realize that the only consistent identifiable epidemiologic precursors for NEC are prematurity and gastrointestinal feeding. Nonetheless, $10 \%$ of affected patients are not premature, and 5-10\% of all patients have not been fed (1).

NEC is a cause of significant morbidity and mortality (20$35 \%$ ) in neonatal intensive care units. NEC may affect $5 \%$ of low-birth weight (LBW) infants, with an even higher incidence among very low birth weight neonates. Based on this information and data available in three data bases, it is estimated that NEC affects 2000-4000 infants in the United States each year (2-6). NEC is assuming a greater proportion of infectious diseaseassociated deaths among LBW infants, and in various centers NEC follows RDS as a cause of neonatal death (4). This latter observation has particular relevance to the current era of neonatology, in which more premature infants receive life-saving treatments with surfactant, antenatal steroids, or high-frequency ventilation. Because the incidence of $L B W$ infants has continued at the same rate for the last 10-15 years, more LBW infants are surviving traditional diseases of prematurity, such as RDS. Because the incidence of NEC is not affected by surfactant therapy, and because NEC is a disease of the surviving premature infant, we can expect an increase in the prevalence of this neonatal illness (2).

\section{OVERVIEW OF DISEASE: CLUES TO PATHOGENESIS}

NEC is a disease that predominantly affects preterm infants recovering from various diseases associated with premature birth. 
As stated earlier, there are no consistent identifiable associated disease states that are more commonly noted in patients with NEC compared with gestational age-matched control infants. This suggests that NEC is related, in part, to immature intestinal function and/or immature gastrointestinal host defense mechanisms.

The pathology of NEC demonstrates mucosal and transmural necrosis and inflammation $(1,2)$. There are associated mucosal ulceration, hemorrhage, and edema in most patients, whereas eosinophilic infiltration and crypt abscesses are noted less frequently. The terminal ileum and proximal colon are most commonly affected, but in the most severe cases the entire bowel (duodenum to rectum) may be involved. Coagulation necrosis (noted in $89 \%$ ) and acute, acute and chronic, or chronic inflammation (noted in $89 \%$ ) may be present in the same area or in different sections of bowel noted in autopsy or surgical pathology specimens. The presence of coagulation necrosis had, in the past, suggested a role for gastrointestinal ischemia as a contributing factor in the pathogenesis of NEC. Nonetheless, similar pathology has been noted in enterotoxemias of developing animals, in pigbel (a disease caused by Clostridium perfringens enterotoxin), and in animal models after exposure to inflammatory cytokines.

The presence of abnormal intestinal gas production, presumably after fermentation of malabsorbed carbohydrates, is noted in $50 \%$ of surgical specimens and in $90 \%$ of documented patients by radiologic methods (1). These grape-sized, gas-filled cysts contain hydrogen, methane, and carbon dioxide gas. Because bacterial fermentation is the only known source of intestinal hydrogen production, a contributing role of the gastrointestinal microflora has been suggested as a factor in the pathogenesis of NEC. Unfortunately, breath hydrogen excretion testing has been difficult to use for the study of neonatal intestinal colonization because of varying degrees of "physiologic" malabsorption and difficulties in performing accurate measurements in premature infants.

NEC may occur during epidemics of disease clustered in place and time (1). Indeed, epidemics of NEC with as many as 20 affected patients in a 3 -week period have been noted in various neonatal intensive care units. When an epidemic occurs, the incidence of all three stages of NEC increases, suggesting that there is an important interaction between the host and the disease that can modify the severity of the illness (Fig. 1). Furthermore, adult personnel in the neonatal intensive care unit often simultaneously demonstrate an increased incidence of gastrointestinal disturbances (nausea, vomiting, diarrhea, or abdominal pain), suggesting the presence of a transmissible agent that may demonstrate different expression in mature and immature hosts. Inasmuch as investigations of most epidemics with standard microbiologic methods have not revealed a consistent (or any) microbiologic agent in affected infants, the study of affected medical staff and other patient contacts may provide important clues regarding the epidemiology of NEC and related gastrointestinal illnesses. Although $20-30 \%$ of patients with NEC may have an associated bacteremia, it is most likely that this represents

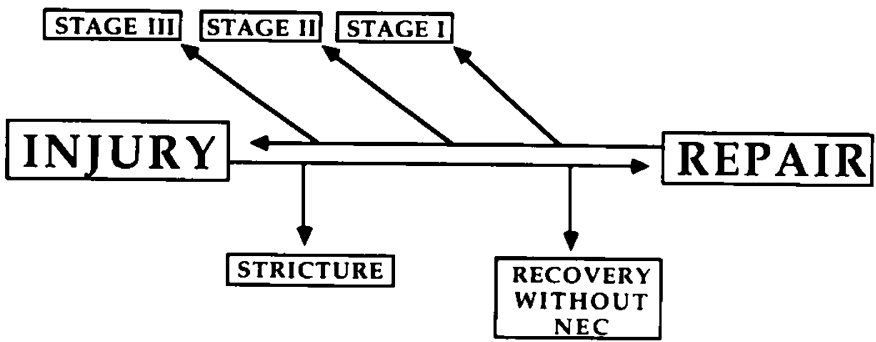

Fig. 1. Interaction of gastrointestinal tissue injury and reparative process along the spectrum of increasing severity of NEC stages and dominance of injury or reparative process. The latter may result in fibrosis and stricture formation or complete healing. bacterial translocation through a previously injured intestinal mucosa rather than the primary initiating event (1).

Recent developments in cell biology and molecular epidemiology have demonstrated the importance of these techniques in investigating diseases such as NEC. In the late 1970 s and early 1980 s, Clostridium difficile was thought to be a causative agent of NEC. Nonetheless, with time and appropriate selection of control infants, $C$. difficile and its potent toxin were discovered in perfectly healthy premature infants. This paradox was recently explained by demonstrating that the immature intestine lacks the brush-border receptor for $C$. difficile toxin A (7).

Rotavirus has been proposed to be another potential initiating agent for NEC. Although rotavirus has been recovered from patients in some epidemics of NEC, this gastrointestinal virus has not been noted in most patients. Standard methods to detect the rotavirus using enzyme-linked assays may be relatively insensitive, as noted by Wilde $e t$ al. (8). These investigators were able to demonstrate the presence of rotavirus by reverse transcriptasepolymerase chain reaction when the enzyme immunoassay was negative. Furthermore, the use of molecular biology has made an outstanding contribution to identifying the responsible microbiologic agent for non A-non B hepatitis (hepatitis C virus), Whipple's disease, and AIDS (human immunodeficiency virus) (9). We must await further use of molecular epidemiology tools in the investigation of epidemics of other diseases that are thought to be caused by an infectious (although presently nonculturable) agent such as NEC.

Recent advances in the prevention of NEC have also led to potential insights to the pathogenesis of the disease. Specifically, the use of glucocorticoids may reduce the incidence of NEC by accelerating gastrointestinal mucosal barrier function or by inhibiting mucosal inflammation (10). The latter mechanism may explain, in part, the beneficial effects of enterally administered IgA-IgG (see below) or expressed human milk (see below) (11, 12).

\section{POSSIBLE ROLE OF INFECTIOUS AGENTS IN PATHOPHYSIOLOGY OF NEC (R. Yolken)}

Several investigators at the conference addressed the possible role of infectious agents as factors or cofactors in the development of some cases of NEC. All the speakers identified similarities between the pathology of NEC and specific enteric infections of humans and animals. Dr. Mitchell Cohen reviewed the pathophysiology of enteric diseases mediated by bacterial toxins and commented that the gastrointestinal pathology of NEC is consistent with the interaction of epithelial cells with bacterial toxins. Although there are a number of bacterial toxins that can cause enteric disease in humans and animals, one that presents similar pathophysiologic findings is the enterotoxin produced by $C$. perfringens type $C$, the causative agent of pigbel (1). Pigbel shares a number of characteristics with NEC, including the development of necrotic lesions in the gastrointestinal tract and the propensity for intestinal perforation. In addition, the development of pigbel requires the interaction of an infectious agent with a number of host-derived gastrointestinal conditions such as malnutrition and the ingestion of foods with protease inhibitory activity. The biology of pigbel may be similar to that of NEC because both diseases may represent the interaction of a number of host-derived and environmental factors. Additional studies should address the detection of bacterial toxins in infants with NEC and should aim to gain better understanding of the host factors that determine the development of disease in affected infants.

Dr. Yolken reviewed the pathophysiology of enteric virus infections. Rotaviruses, adenoviruses, and other recognized enteropathogenic viruses generally infect epithelial cells, resulting in cytolysis and epithelial cell destruction. In older infants, this cellular destruction produces increased intestinal fluid output and the development of diarrhea. However, in the case of neo- 
nates who may be unable to respond to infection with increased fluid output, such cellular destruction may have other consequences including epithelial cell necrosis or intestinal perforation. Furthermore, in older infants, the pathogenic consequences of enteric virus replication are limited by the fact that the necrotic epithelial cells are replaced by regenerated epithelial cells arising from the intestinal crypts. It is likely that the regenerative capacity of high-risk neonates is compromised and thus that virusinduced intestinal cell destruction may not be counterbalanced by sufficient cellular regeneration.

In the past, the ability to study viral infections of the gastrointestinal tract has been limited by the unavailability of sensitive methods for viral detection (8). The recent developments of sensitive polymerase chain reaction assays and other new molecular diagnostic tests for the accurate detection of enteric pathogens represent important new tools for the efficient study of enteric pathogens in infants with NEC. Such assays can also be used to determine the prevalence of infection in contacts and controls and the molecular similarity between viruses isolated in different infants. The application of this methodology could lead to an increased understanding of the role of specific pathogens in the epidemiology and pathophysiology of NEC.

Dr. Yolken also discussed alterations of anti-infection host factors that might reduce the ability of a neonate to respond to enteric infections. In addition to the cellular and humoral components of the immune system (see below), intestinal mucins may play an important role in the modulation of enteric infections. Dr. Yolken presented data indicating that rotaviruses bind to intestinal mucins and that mucins can inhibit viral replication in vitro and in animal model systems. Dr. Cohen pointed out that mucins can also bind enteric bacteria and their toxins. Intestinal mucins may plan an important role in the host's ability to modulate the intestinal replication of pathogenic agents. Correspondingly, alterations in the mucin barrier related to prematurity, diet, or alterations in the intestinal microenvironment may make premature infants more susceptible to NEC. Mucins with antiviral activity are also found in human milk and are thus part of the diet of breast-fed infants. The commercial development of mucins suitable for feeding to non-breast-fed infants may thus be a potential therapeutic modality for the modulation of enteric infections in this population.

Dr. Kinney reviewed previous studies directed at elucidating the epidemiology of NEC. She presented data indicating that some outbreaks of NEC are associated with a temporal pattern that is consistent with endemic or epidemic infection. Furthermore, some of the epidemics have been associated with the recovery of recognized enteric viruses in the affected infants. In addition, some outbreaks are simultaneously associated with enteric infection and diarrheal disease in older infants and in medical staff. The epidemiologic and microbiologic analysis of disease patterns in the contacts of neonates with NEC may thus be an important step in elucidating the pathophysiology of NEC itself. Because preterm infants may not be able to respond to enteric infection by the development of increased intestinal fluid output and recognizable diarrhea, the presence of a transmissible agent within a neonatal nursery may not be suspected and the proper studies may not be performed. Furthermore, because ill staff may serve as a source of infection for the neonates, the occurrence of outbreaks of gastrointestinal illness in the medical or nursing staff may herald the need for the institution of infection control measures. It should be noted that infections with enteric agents do not appear to be necessary or sufficient for the development of all cases of NEC. Thus, some cases of NEC do not follow an epidemiologic pattern consistent with traditional gastrointestinal infection and appear to be unrelated to infections with known enteric agents. Similarly, even during outbreaks, some infants with documented enteric infection do not develop NEC. It thus appears likely that, as in the case of pigbel described above, host factors play an important role in the development of NEC. Nonetheless, the study of the role of infectious agents may shed light on the pathogenesis of NEC and may lead to the development of new modalities for the prevention of many cases of this disease.

\section{GUT IMMUNOLOGY (R. M. Goldblum)}

There are several reasons to consider the development of local immunity of the gastrointestinal tract in attempts to understand the pathogenesis of NEC. These might be stated as two related hypotheses: $l$ ) because NEC is largely a disease of the immature infant, immaturity of the local host defenses of the gut is a factor; and 2) immaturity of gut immunity causes unique susceptibility to specific infections and/or poorly regulated inflammatory responses, both of which can cause localized injury of the gut.

Drs. Kagnoff and Goldblum presented overviews of our current knowledge of the function of the immune system of the gut and the state of development of this system in the human neonate, respectively. Gut immunity, like systemic immunity, is the product of several interrelated components. Nonspecific or innate resistance is mediated by factors such as $\mathrm{pH}$, enzyme, mucins, epithelial barriers, gut motility, and secreted nonspecific antimicrobial factors such as lactoferrin and lysozyme. All these factors may alter microbial and antigenic density in the gut. The limited data available suggest that the quantities or activities of each of these factors are lower in the premature infant than in the more mature infant or the adult.

The immunologically specific responses in the gut can be divided into the $B$ cell (humoral immune) and $T$ cell (cellmediated) compartments. Humoral immune responses in the gut are largely initiated in specialized lymphoid aggregates called Peyer's patches, which are concentrated in the small intestine. Here, the contents of the intestinal lumen, especially particulate antigens such as microorganisms, are sampled by specialized epithelial cells and presented to subjacent populations of $T$ and B lymphocytes and macrophages. A well-demonstrated outcome of these events is the activation and emigration of B lymphocytes that are largely committed to making specific antibodies of the IgA class. The Peyer's patches may also be a source of specific $T$ cells for the gut, but this pathway is less well defined. Both $T$ and B cells can be identified in Peyer's patches from about 19 weeks of gestation in the human fetus. In rodents, however, these lymphoid cells are not present in the Peyer's patches until after birth. Thus, these common experimental animal models may have limited usefulness in immunologic studies of human gut maturity.

The activated B cells that leave the Peyer's patches eventually migrate through intermediate lymphoid organs and lymphatic and blood vessels before homing to sites along mucosal surfaces, particularly the submucosa of the intestinal tract. The homing process is apparently mediated by the interaction of receptorligand pairs that are expressed on the surface of the $B$ cell and vascular endothelium. Normally, very few B cells are present in the lamina propria of the gut until several weeks after birth, presumably because of the lack of antigenic stimulation in utero. Nonetheless, oral immunization investigations have shown that the system for generating specific immune responses in the gut is present at birth, even in the premature infant. However, it is not known whether the repertoire of antibodies that can be made by the immature infant are comparable to those of the older infant or adult.

The major T lymphocyte populations within the gut are in the submucosa, and those between the epithelial cells are the intraepithelial lymphocytes (IEL). The origin of IEL and their developmental patterns are not well understood. Cells that are of thymic origin, as well as $T$ cells that mature outside the thymus, are probably involved. Both the lamina propria and intraepithelial T cells are present from 14 to 16 weeks of gestation. However, the composition of these $T$ cells may be quite different from that of the adult cells, as indicated by the surface phenotypes. It is difficult to interpret these findings, because the normal functions 
of the intestinal T cells, particularly the IEL, have not been defined. However, it seems likely that these cells destroy injured or infected epithelial cells. Inadequate regulation of this process could have obvious impact on the pathogenesis of intestinal ulcerative diseases like NEC.

An area of evolving interest is the interactions between the various cell types in the intestine, particularly through signaling molecules or cytokines that allow two-way communication between $\mathrm{T}$ and $\mathrm{B}$ lymphocytes, stromal elements, and epithelial cells. A well-integrated communication system is likely to be critical for maintaining the adequate function of the gut and protecting it without causing excessive tissue injury.

Dr. Goldman presented an overview of the immunologic factors in human milk. The evolving concept is that the nursing infant receives an array of nonspecific and specific immune factors from its mother's milk that may offer protection during the postnatal period when the infant's immune system is developing. In addition, it is possible that human milk feeding enhances the rate of development of the infant's own local immunity in light of recent findings of immunomodulators in human milk. Dr. Goldman also offered evidence that human milk may protect the infant's intestinal tract without causing inflammatory reactions; alternatively, human milk may even suppress inflammation. Both the passive protection and hypothesized anti-inflammatory activities of human milk may provide a partial explanation for the finding that premature infants fed completely or partially with human milk have a lower incidence of NEC.

The concept that IgA, the predominant immunoglobulin class in human milk and endogenous intestinal secretions, may inhibit inflammation was also considered by Dr. Eibl. She described recent experiments in which the release of proinflammatory cytokines from blood monocytes was inhibited by $\mathrm{IgG}$ and serum-type IgA. The effect of IgA and IgG on activation of monocytes by various stimuli has been evaluated. With a few exceptions, the anti-inflammatory effects of both classes of immunoglobulins in this system might be explained by neutralization of the antigenic stimuli by specific antibodies in the immunoglobulin preparations. The possibility that immunoglobulins, especially IgA, may have a broader anti-inflammatory effect remains interesting but unproven. Dr. Eibl's clinical trials suggesting that feeding a mixture of $\operatorname{IgG}$ and $\operatorname{IgA}$ reduces the incidence of NEC are consistent with this possibility (11). Confirmation of these findings awaits the results of additional blinded, placebo-controlled trials.

Dr. Israel reviewed her studies that suggest that in the human fetus, as in newborn rodents, enterocytes transiently express a receptor that can bind IgG. The human receptor has many of the same characteristics of the Fc $\gamma$ receptor found postnatally in the rodent gut. The rodent receptor mediates the transport of maternal milk IgG from the gut lumen into the circulation. The functional significance of such a receptor in the human fetus remains in question, inasmuch as amniotic fluid, the potential source of $\mathrm{IgG}$ in the gut lumen during gestation, contains very low concentrations of $I g G$.

Discussions during this session emphasized several points. First, our understanding of the normal development of the immune system in the human gut is deficient. A major gap exists in our data between 20 weeks and near term gestation, the peak period for NEC in the preterm infant, largely because of the very limited availability of clinical material. Our current understanding of intestinal immune development lacks detail throughout gestation, particularly with respect to the function of various immune cells. For instance, one common phenotype among the intraepithelial lymphocytes from early fetuses is very rare in infants and adults; the physiologic and pathologic significance of these cells in the premature infant has not been explored. In addition, more studies are needed to explore the hypothesis that fetal and neonatal gut immune function are regulated by amniotic fluid and human milk, the fluids that normally bathe the gut lumen. These fluids and their constituents represent potential therapeutic agents for altering the interaction between the immune and epithelial cells, thereby providing potential approaches for preventing or treating NEC.

\section{INTESTINAL INFLAMMATION (C. Wilson)}

This session addressed the roles of inflammatory mediators, including cytokines and lipid-derived mediators, and of inflammatory cells in the pathogenesis of NEC. The hemorrhagic intestinal necrosis that characterizes NEC has also been observed after ischemia and after systemic administration of proinflammatory cytokines, such as tumor necrosis factor- $\alpha$ (TNF). Ischemia, particularly when subsequently accompanied by partial or complete restoration of blood flow, can induce or act as a potent cofactor for the production of cytokines and other proinflammatory mediators (13). Thus, the nature of the injury observed in NEC cannot be used to argue for or against hypoxia/ischemia as an inciting event in this disease process and is consistent with a multietiology or multifactorial syndrome of intestinal injury. This is illustrated by certain similarities in results from animal models, which use disparate inciting agents for intestinal injury.

Dr. Chang provided an overview of potential mechanisms of intestinal inflammation. He also showed evidence that cytokines, such as IL-1 (a product of many cell types including epithelial cells, macrophages, and endothelial cells) and IL-3 (a product of mast cells and $T$ lymphocytes) can act on intestinal tissue to induce secretion of anions, acting in part through prostaglandin $E_{2}$ and cAMP produced by cells in the subepithelium.

Drs. Hsueh and Caplan presented both clinical data and data from their experimental model of NEC in juvenile rats, which support roles for the cytokine TNF and the lipid-derived mediator platelet-activating factor (PAF) in NEC (12). In humans with overt NEC, these workers have found that concentrations of PAF and TNF are significantly increased in the plasma compared with age-matched controls. Furthermore, the levels of the enzyme that catabolizes PAF, acetylhydrolyase, are reciprocally depressed in affected patients. Using in situ hybridization to detect TNF mRNA in viable tissue at the margins of necrotic intestine removed surgically form infants with NEC, they found that neither intestinal lymphocytes nor submucosal macrophages contained TNF mRNA. The only cells containing TNF mRNA were the intestinal Paneth cells, which are known to constitutively express this mRNA. Thus, if representative of the affected intestine in evolving NEC, the source of increased circulating TNF in patients with this disease is not evident. It is possible that Paneth cells may be induced to translate and secrete TNF in NEC, or to do so in amounts sufficient to be detectable in the circulation, although this seems less likely. It is also possible that circulating monocytes or lymphocytes could be the source of the excess TNF, but this remains to be determined; such a possibility is consistent with the observation in animal models that systemically injected TNF can induce local ischemic injury in the intestine in a pattern similar to that in NEC.

Supporting the relevance of the excess TNF and PAF observed in affected patients are studies by Drs. Hsueh and Caplan and other groups who work with rodent models. Intravascular injection of TNF, bacterial lipopolysaccharide, and PAF can independently induce ischemia-like intestinal necrosis resembling NEC. Furthermore, each of these agents if used in combination with one of the others or with hypoxia (at least in the case of lipopolysaccharide) produces greater injury than when used alone at doses inducing partial injury. PAF antagonists attenuate bowel necrosis and many of the other features of injury (e.g. metabolic acidosis) of this model. This has led these investigators to suggest that PAF may be a critical downstream mediator of the effects of the other agents.

Dr. Wilson provided an overview of the events initiating production of TNF and other proinflammatory cytokines, the cells that produce these mediators, and the mechanisms by which 
they contribute to endothelial injury and inflammatory cell recruitment. In NEC, potential sources of TNF include intestinal mast cells (which contain preformed TNF and can thus release this mediator immediately upon degranulation), intestinal macrophages and $\mathrm{T}$ cells, circulating leukocytes, and potentially mucosal Paneth cells. A variety of epithelial cells, cells present in the submucosa, and endothelial cells are capable of producing IL-1 and chemotactic cytokines such as IL-8. Caveats were raised regarding the methods by which their production in vivo and contributory role as mediators can be determined: 1) These mediators in physiologic circumstances act primarily locally and are rapidly cleared from the circulation. Assays that measure circulating concentrations may fail to detect them even if they play a role in disease. Assays of TNF protein and mRNA on lysates of cells that produce these mediators may be necessary to detect their production, for example in tissues or in circulating leukocytes. The lack of detectable TNF mRNA in intestinal cells other than Paneth cells suggests that examination of circulating leukocytes for TNF mRNA and protein might be revealing. 2) A considerable amount of IL-1 ( $\alpha$ to a greater extent than $\beta$ ) remains cell associated. Thus, IL-1 may only be detected by these means. 3) Cytokines and lipid-derived mediators commonly act in an additive or synergistic manner. Thus, inhibition of experimental disease by antagonism of one mediator is not evidence that this is the ultimate or downstream mediator of disease. Conversely, these results suggest that even if multiple mediators are involved, antagonism of any one may be sufficient to ameliorate disease.

Dr. Clark presented evidence that ischemia is not necessary to produce intestinal mediator release and injury. In rabbits, intraluminal injection of casein and calcium at an acidic $\mathrm{pH}$ results in an injury that reproduces many of the pathologic manifestations of NEC. This is associated with increased intestinal permeability, a rapid release of histamine, and increased release of eicosanoids (prostaglandins and leukotriene $\mathrm{B}_{4}$ ). Intestinal injury and eicosanoid release are retarded by superoxide dismutase, suggesting a role for reactive oxygen metabolites in this nonischemic injury model. Because this injury occurs in isolated intestinal loops and develops before the influx of inflammatory cells, it is suggested that local cells are capable of releasing mediators sufficient to initiate this injury. Whether this is an accurate reflection of what occurs in NEC in human neonates and which cells might be involved in the production of these mediators remain undetermined.

\section{NUTRITION AND NEC (R. M. Kliegman)}

Enteral alimentation is thought to contribute to the pathogenesis of NEC because $90-95 \%$ of patients become ill after receiving milk (usually formula) feedings (1). Despite this relationship, the mechanism(s) involved has (have) not been defined. Although most neonatologists agree that rapid feeding schedules place the premature infant at risk for NEC, the exact rate of formula increment that predisposes infants to NEC has not been identified.

Milk feedings may produce "nutritionally induced inflammation," which may be caused by several hypothetical mechanisms (2). First, malabsorbed carbohydrate may serve as a substrate for the intestinal microbiologic flora, which then ferment lactose to hydrogen gas and short-chain fatty acids. The former may be responsible for the gaseous distention and pneumatosis intestinalis, whereas the short-chain fatty acids may (if in high concentration) be toxic to the intestinal epithelial cell. Malabsorbed long-chain fatty acids, oleic acid in particular, may also produce increased intestinal permeability and hemorrhagic necrosis if presented to the distal ileum in high quantities (see below). Finally, as commented above, undigested casein may contribute to intestinal inflammation and injury.

The onset of intestinal alimentation among preterm infants has been reported to increase the proliferation of enteric bacteria and to produce a transient endotoxemia. Locally elevated levels of cytokines and lipid mediators of inflammation may follow and initiate a nutritionally induced inflammation. Without an appropriate balance of anti-inflammatory mechanisms, the inciting inflammation may create a vicious cycle of events propagating the injury thus leading to NEC (Figs. 1 and 2).

Alterations of host defense or intestinal injury repair mechanisms may predispose to NEC and tip the balance toward unopposed inflammation. If there has been a significant period (days) of no enteral feedings, mucosal (villous) atrophy may intervene. In the absence of local trophic nutrients that support and stimulate mucosal growth (directly and indirectly by stimulating "gut hormone" production), the resulting villous atrophy may predispose the premature infant to endotoxin or bacterial translocation with subsequent intestinal or systemic inflammation. Investigations among adult patients in the postoperative period have emphasized the importance of early enteral feedings as a safe route for nutrition support and as a means to reduce the complications associated with endotoxin or bacterial translocation. Multiple studies have demonstrated the nutritional benefits of early "hypocaloric-minimal enteral feedings" in premature infants (14). Infants receiving hypocaloric minimal feedings have fewer episodes of feeding intolerance, gain weight sooner, and have a lower incidence of osteopenia of prematurity and cholestatic liver disease without influencing the incidence of NEC.

Expressed natural milk has been demonstrated to reduce the incidence of NEC in an animal model and among preterm infants. Expressed human milk has many potentially advantageous properties that may reduce the risk for NEC (12). Expressed human milk contains various specific cellular and humoral components of immune defense, in addition to many nonspecific host defense factors.

Expressed milk immune cells have been thought to protect newborn animals against NEC. However, there is a paucity of information about the specific (cellular or humoral) protective factors in expressed human milk. The presence of IgA in expressed milk may have a role in immune protection as demonstrated in Dr. Eibl's investigation of supplementing feedings with IgA-IgG. Premature infants who received IgA-IgG demonstrated a reduced incidence of NEC despite regaining birth weight more rapidly than infants not receiving $\operatorname{IgA}-\mathrm{IgG}$. More rapid feeding traditionally predisposes to NEC; however, the beneficial effects of IgA-IgG supplementation may have attenuated the risks of rapid feeding, possibly by its anti-inflammatory or microbiologic neutralizing properties. Additional anti-inflammatory factors in expressed milk may include PAF acetylhydrolase, vitamin $\mathrm{E}, \beta$ carotene, and ascorbic acid.

Expressed human milk may have nonimmunologic, non-host defense properties that are beneficial to preterm infants and thus reduce the risk of NEC. Expressed human milk lipids may be less toxic to the intestinal epithelium than those in commercial formula. Specific nutrients (glutamine, nucleotides) may be beneficial to gastrointestinal cell metabolism and function. Furthermore, expressed human milk feeding results in an attenuated increase in postprandial mesenteric blood flow, possibly requiring a reduced intestinal hyperemia after a feeding. Finally, expressed human milk has many growth factors (IGF-I, epidermal growth factor) and hormones (thyroid) that may directly improve gastrointestinal function and maturity.

There remain many unanswered questions related to the important clues suggesting a role of enteral nutrition in the pathogenesis of NEC. When does nutritionally induced inflammation represent a physiologic response to normal feedings (e.g. tissue repair and recovery), and what alterations of host factors are responsible for an unbalanced inflammatory response and possibly NEC? What is the role of malabsorption (carbohydrate, triglycerides-long-chain fatty acids, casein) or altered intestinal motility in the pathogenesis of NEC? How can neonatologists actively reduce the risk of NEC by improving our understanding 


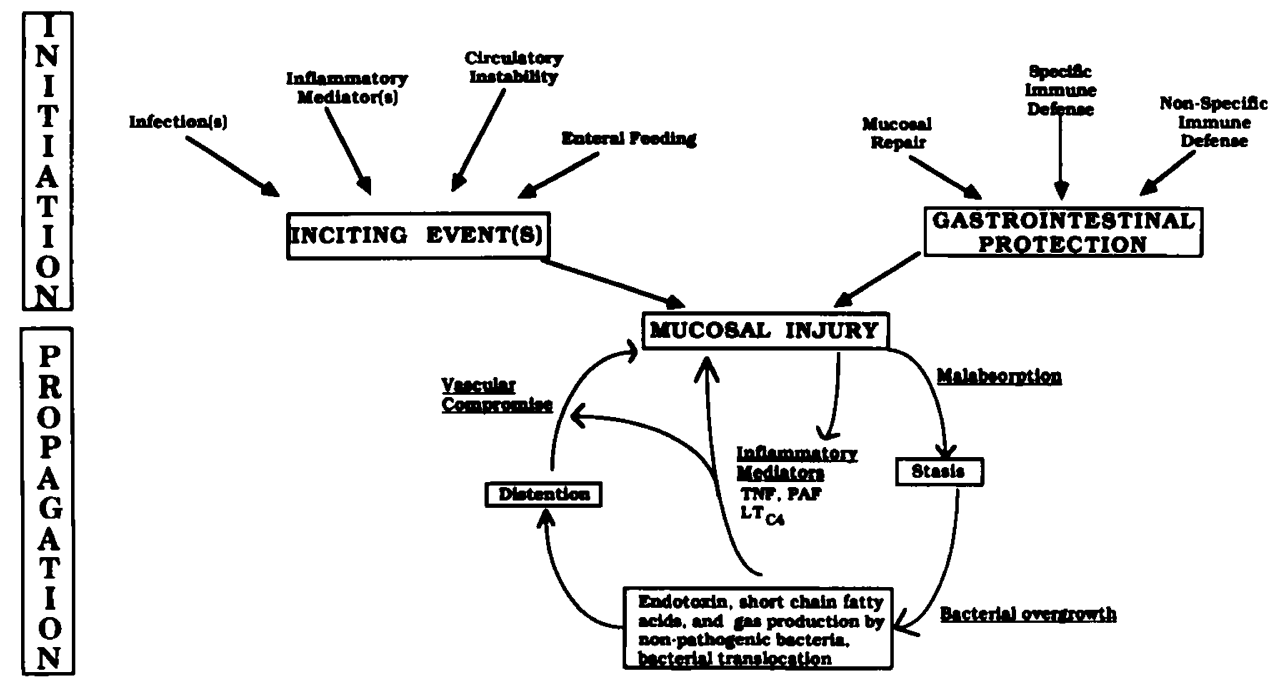

Fig. 2. Relationship of initiating events that relate to tissue injury (inciting events $v s$ poor host protective mechanisms) and secondary or propagating events in NEC. Specific initiating events may be balanced by reparative processes, or if unbalanced may lead to a series of events that produce secondary alterations of gastrointestinal function that contribute to NEC.

about intestinal physiology and subsequently altering our current feeding practices?

\section{GASTROINTESTINAL CIRCULATION (K. Crissinger)}

Work performed over the last several years has focused on establishing a better understanding of circulatory physiology and pathophysiology in the developing intestine. Because of the high metabolic demands of growth, intestine from the young mammal has a higher baseline level of blood flow and oxygen consumption compared with intestine from older animals. To cope with normal physiologic metabolic demands, developing intestine can respond by either increasing blood flow, increasing oxygen extraction, or increasing both. If further ischemic and/or hypoxic stresses exceed the intestine's capacity to maintain tissue oxygenation, intestinal injury may result (15-17).

Intrinsic regulatory capacity in developing intestine has been evaluated by perturbations of arterial pressure (capacity for autoregulation, reactive hyperemia), venous pressure (venous pressure hypertension), arterial oxygen concentration (hypoxia), and increased metabolic demand (postprandial hyperemia). In the majority of studies, investigators have demonstrated significant developmental differences in the capacity for regulation of intestinal blood flow and oxygen consumption. In general, the intestine from younger animals had a limited capacity for increasing intestinal blood flow in response to a cardiovascular stress, although it is frequently able to compensate by a dramatic increase in oxygen extraction. This capacity for increasing oxygen extraction, however, can also be overwhelmed, thus subjecting developing intestine to tissue hypoxia.

Two examples of a reduced capacity for intrinsic regulation of intestinal blood flow are the responses to arterial pressure reduction and to feeding. Intestine from 35-day-old swine demonstrates vasodilation after arterial pressure reduction; however, this response is incomplete and blood flow falls in response to hypotension. This flow reduction is countered by a rise in arteriovenous $\mathrm{O}_{2}$ difference $\left[(\mathrm{a}-\mathrm{v}) \mathrm{O}_{2}\right]$ of nearly equal magnitude, such that intestinal oxygenation is not compromised until arterial pressure is reduced below $50 \%$ of baseline. The rise in $(\mathrm{a}-\mathrm{v}) \mathrm{O}_{2}$ is mediated, in part, by an increase in the perfused capillary density, as evidenced by an increase in capillary filtration coefficient $\left(\mathrm{K}_{f, 0}\right)$ noted in response to flow reduction in older subjects. In addition, the rise in (a-v) $\mathrm{O}_{2}$ may reflect stabilization of the capillary-cell $\mathrm{PO}_{2}$ gradient, achieved by modest reduction in cellular $\mathrm{PO}_{2}$. Intestine from 3-day-old swine, in contrast to that from older subjects, fails to demonstrate vasodilation in response to arterial pressure reduction; indeed, vascular resistance actually increases after pressure reduction, so that reductions in blood flow are significantly greater than those noted in older subjects. $K_{f, 0}$ increases in response to flow reduction, albeit to a modest degree and to an extent significantly less than that noted in older subjects. Thus, the rise in $(\mathrm{a}-\mathrm{v}) \mathrm{O}_{2}$ noted after flow reduction is relatively small and not of sufficient magnitude to preserve intestinal oxygenation. This compromise of intestinal oxygenation in intestine from 3-day-old swine occurs in response to even modest degrees of arterial pressure reduction. The lack of effective vasodilation or capillary recruitment in younger animals is not simply the consequence of the relative state of vasodilation that characterizes this circulation at rest; a significant reduction in vascular resistance and increase in $K_{f, 0}$ occur in response to $\beta$ agonist infusion. Instead, it appears that the intestinal microcirculation is relatively insensitive to the oxidative demands of the parenchyma.

Investigation of the hemodynamic and oxygenation responses to formula feeding in developing intestine has demonstrated that 1-day-old intestine from piglets who have never been allowed to nurse (4-8 hours of age) is incapable of increasing total wall intestinal blood flow in response to feeding. This is in contrast to 3-day-, 2-week-, and 1-month-old animals, which do increase intestinal blood flow in response to feeding. One-day-old intestine is, however, able to significantly increase oxygen extraction so that consumption during feeding is not compromised during this normal physiologic stress. This points out the importance of knowing the response of both blood flow and oxygen extraction when speculating about the effects of decreasing intestinal blood flow in developing intestine. Despite the maintenance of oxygen uptake with feeding in 1-day-old intestine, the capacity for increasing oxygen extraction is near maximal, so that additional hypoxic stresses may lead to tissue hypoxia.

Additional work attempting to demonstrate a link between this increased susceptibility to tissue hypoxia has used a model of intestinal ischemia and reperfusion. Indeed, when 1-day-old piglet intestine is subjected to 60 minutes of ischemia and 60 minutes of reperfusion in the presence of a predigested and bile acid-solubilized cow milk-based artificial pig milk formula, significantly larger increases in mucosal permeability to ${ }^{51} \mathrm{Cr}$ EDTA occur during reperfusion in 1-day-olds compared with all older age groups.

The nutrient component of formula responsible for this developmental increase in mucosal permeability may be in the lipid fraction, inasmuch as results similar to those induced by formula perfusion occur with lipid perfusion after an hour of 
ischemia and an hour of reperfusion in 1-day-old intestine. Perfusion with glucose or protein does not lead to increased mucosal permeability. Furthermore, the increase can be abolished by delipiding the formula. This difference in permeability as a function of nutrient component does not occur in 1-monthold intestine. Interestingly, lipid perfusion also leads to increases in mucosal permeability during control periods, in the absence of ischemia and reperfusion, in 1-day-old intestine but does not occur in 1-month-old intestine.

Finally, it has also been demonstrated that direct perfusion of developing piglet intestine with predigested and bile acid-solubilized premature infant formula causes significantly greater injury compared with standard term formula even in the absence of ischemia and reperfusion. Removing the lipid from the formula dramatically ameliorates this injury. The additive effects of ischemia and reperfusion with this premature formula preparation result in hemorrhagic and necrotic tissue.

In summary, the baseline high rate of oxygen utilization renders newborn gut more susceptible to tissue hypoxia in the face of superimposed hypoxic stresses, in that the intestinal circulation from young subjects appears relatively incapable of increasing oxygen transport in response to states of impaired oxygen availability. Additionally, young intestine undergoes significantly more injury in response to nutrient perfusion, specifically by the lipid fraction. Furthermore, the combination of feeding and ischemia may lead to the development of a NEC-like disease in 1-day-old piglet intestine. Thus, beneficial therapy aimed at preventing NEC may result from efforts to decrease the metabolic demands placed on the intestine, as well as providing long-chain fats (necessary for membrane and neurologic development) in a form that is absorbed by developing intestine but that does not result in intestinal injury.

\section{DYNAMIC STATUS OF BALANCE BETWEEN INTESTINAL INJURY AND REPAIR: BALANCING HOST WITH EXOGENOUS FACTORS}

There are many models of human gastrointestinal disease that are more severe in the younger age group and that are modified by various host and exogenous dietary practices. Pigbel is more severe in children than adults; the disease in both age groups follows a traditional pig feast. The disease is caused by an enterotoxemia due to a $C$. perfringins toxin but is made more severe in the presence of reduced intestinal trypsin activity. The latter may be caused by associated malnutrition with reduced pancreatic function or with the ingestion of potatoes, which contain protease inhibitors. Both conditions may reduce the neutralization of toxin in the intestinal lumen and move the balance of host defense versus disease toward enhanced toxin action. Pigbel may be successfully treated with passive immunization and prevented with active immunization with toxoid.

NEC may represent an imbalance between a continuing physiologic cycle of gastrointestinal tissue injury and repair. Indeed, pathologic specimens from patients with NEC demonstrate reparative processes juxtaposed to areas of acute and chronic inflammation. As the balance moves further from tissue healing and repair, the various stages of NEC may become manifest (Fig. 1). Many endogenous or exogenous factors may move the balance toward disease (Fig. 2). Injurious factors may include infectious agents, nutrient induced inflammation, and microcirculation instability, whereas the repair process may be attenuated by immaturity or absence of anti-inflammatory factors present in expressed human milk. Once initiated, mucosal injury can lead to a continuous propagating cycle of injury, inflammation, and vascular compromise (Fig. 2). The relative role of each of these individual factors in the pathogenesis or prevention of NEC has yet to be determined.

\section{FUTURE DIRECTIONS}

It became quite evident to the multidisciplinary group of basic scientists and clinical investigators that there are wide gaps in our understanding of neonatal gastrointestinal physiology and pathophysiology. Considering the large numbers of infants who develop NEC each year and the significant morbidity and mortality associated with this illness, NEC warrants further investigation. The model outlined in Figure 2 helps to reconcile many divergent theories related to NEC. Furthermore, because NEC presents within a broad spectrum of gastrointestinal severity, it was suggested that a clinical classification as proposed previously and strict diagnostic staging criteria be used to avoid confusion about a disease with a multifactorial basis. Specific areas that are amenable to investigation were discussed repeatedly at the conference and include the following:

- The investigation of normal gastrointestinal physiology and pathophysiology among term and preterm infants in the areas of host protection, blood flow, endothelial integrity and mucosal function should be linked to randomized trials to reduce the incidence of NEC. Promising preventive interventions include expressed human milk, enteral administration of IgAIgG or mucins, and postnatal steroid administration. Future randomized controlled trials must be integrated with basic science protocols to better define the mechanism(s) of protection. Future preventive studies may include various feeding protocols and administration of pentoxyphylline or PAF inhibitors.

- The transition from the fasted to the fed state is a specific area that needs further investigation. The potentials for nutritionally induced inflammation, the role of bacterial fermentation with intestinal gaseous distention, and the proliferation of colonizing bacteria with the release of endotoxin and various cytokines need to be determined. The modulation of gut flora by intestinal immune response mechanisms is a particularly exciting area for further investigation.

- The ability to prospectively apply molecular epidemiology tools to the study of NEC (18) should be investigated. Such methods could include the development of a tissue bank of carefully resected and preserved surgical pathology specimens. This is analogous to tumor banks that serve as a resource to molecular oncologists, who use them to probe for the presence of new discovered oncogenes. Such surgical specimens may provide a valuable resource for the investigation of the presence or signs of specific microbial agents, enterotoxins, or mediators induced by such enterotoxins or endotoxins. For example, in situ hybridization may serve to identify microbial agents, inflammatory mediators, enzymes playing a role in the production or metabolism of inflammatory mediators, or long-lived alterations in the vascular endothelium or mucosal and submucosal tissue related to the presence of such mediators. Investigations such as this may productively use combinations of immunocytochemistry, immunoelectronmicroscopy, and in situ hybridization to detect messenger RNA. The advantage of these techniques is that they not only identify specific mediators, but can localize their effects to the producing cell or the target cell. Furthermore, the prospective banking of serial collections of plasma, circulating leukocytes, urine, or cells collected from stool or intestinal secretions can serve as a resource for identification of potential pathogens, demonstration of the excretion of stable metabolites of inflammatory mediators, identification of cell type activation, or identification of expression (mRNA) of cytokines in exfoliated enterocytes or excreted inflammatory cells. Stool can also be probed for molecular markers to identify unculturable but potential pathogenic organisms or toxins. These methods can be applied to affected and unaffected adult contacts and to endemic and epidemic cases if the samples are collected prospectively from affected and age-matched unaffected infants.

- The reported 10-fold difference in the incidence of NEC be- 
tween neonatal centers may hold important clues to the nosocomial nature of this disease (6). Such a 10 -fold difference in outcome related to carotid artery surgery or coronary artery bypass operations has produced important practice guidelines from the Agency for Health Care Policy Research. Similar epidemiologic investigations should be performed for NEC. Now that many of the previously purported risk factors have not proven to be important precursors to the development of $\mathrm{NEC}$, we are in a much better position to ask new and more specific questions related to the epidemiology of NEC.

- Although NEC can be readily diagnosed by plain roentgenography and pathologic examination of tissue, participants felt that new and more specific diagnostic approaches to this disease are needed. There is a particular need for a marker that will detect early stages of the disease process and that might be used to monitor the efficacy of therapeutic interventions early in the course of the illness. Furthermore, specific diagnostic tools would also provide insight into normal gastrointestinal physiology and host defense mechanism. Tools such as molecular biology probes for antigen or genome detection of infectious agents, assays to detect stable metabolites of cytokines, and Doppler ultrasound flow studies of the mesenteric circulation are being or are ready to be applied to this task (18). Newer methods may be developed or modified for use in the neonate. An example would be the development of nuclear magnetic resonance spectroscopy through an indwelling intraintestinal catheter to study the adequacy of enterocyte oxidative metabolism.

- The need for animal models that replicate the human disease was emphasized. Such studies should occur in parallel with human studies. There is a particular need for animal investigations that can address multifactorial etiologies (i.e. prematurity, infection, nutrition, blood flow, and interactions between these potential contributing factors).

- Epidemiologists, basic scientists, and clinical investigators working with the multicenter neonatal network would have an exciting opportunity to address these investigations with a shortened time frame and with rigorous broad-based multidisciplinary scientific methods (6). Working together, the investigators and the network's centers would have a large, coordinated patient population to accomplish research activities in a shorter time than if the research agenda was approached one neonatal center at a time. The ability to bring a multidisciplinary research team together with a large patient population would expedite research and save money. In this context, prospective studies need to be designed that will allow for the proper collection of specimens and availability of relevant specimens to interested investigators.

Acknowledgment. The authors thank Charlotte Catz, M.D.; Gilman Graves, M.D.; Ephraim Levin, M.D.; Linda Wright, M.D.; and Sumner Yaffe, M.D., of the National Institute of Child Health and Human Development for planning, sponsoring, and coordinating this conference. We also thank the following basic scientists and clinical investigators who participated in the conference for leading the excellent scientific discussions. Conference Organizers: Robert M. Kliegman, M.D., Medical College of Wisconsin, Children's Hospital of Wisconsin; W. Allan Walker, M.D., Harvard Medical School, Children's Hospital; Robert H. Yolken, M.D., The Johns Hopkins Hospital; Randall M. Goldblum, M.D., University of Texas Medical Center, Galveston; Christopher B. Wilson, M.D., University of Washington; Karen D. Crissinger, M.D., Ph.D., Louisiana State
University Medical Center; and Ephraim Levin, M.D., National Institute of Child Health and Human Development. Section Chairs: Infectious Agents, Robert H. Yolken, M.D.; Gut Immunology, Randall M. Goldblum, M.D.; Intestinal Inflammation, Christopher B. Wilson, M.D.; Nutrition, Robert M. Kliegman, M.D.; and Gastrointestinal Circulation, Karen D. Crissinger, M.D., Ph.D. Presenters and Participants: Susan S. Baker, M.D., Ph.D., University of Massachusetts Medical Center; Michael Caplan, M.D., Evanston Hospital, Northwestern Hospital; Eugene Chang, M.D., University of Chicago; David A. Clark, M.D., Louisiana State University; Mitch Cohan, M.D., Children's Hospital Medical Center; Martha M. Eibl, M.D., Institute of Immunology, Vienna, Austria; Armond S. Goldman, M.D., University of Texas Medical Center, Galveston; Neil Granger, Ph.D., Louisiana State University Medical Center; Wei Hsueh, M.D., Ph.D., Northwestern University School of Medicine, Children's Memorial Hospital; Esther J. Israel, M.D., Massachusetts General Hospital; Martin Kagnoff, M.D., University of California, San Diego; Janet Kinney, M.D., The Children's Mercy Hospital, Kansas City, MO; Philip T. Nowicki, M.D., Children's Hospital, Ohio State University; Philip M. Sherman, M.D., Hospital for Sick Children, Toronto, Ontario; E. Richard Stiehm, M.D., UCLA School of Medicine; and John Udall, Jr., M.D., Ph.D., University of Arizona.

\section{REFERENCES}

1. Kliegman RM, Fanaroff AA 1984 Necrotizing enterocolitis. N Engl J Med 310:1093-1103

2. Kliegman RM 1990 Neonatal necrotizing enterocolitis: bridging the basic science with the clinical disease. J Pediatr 117:833-834

3. Palmer SR, Biffin A, Gamsu HR 1989 Outcome of neonatal necrotising enterocolitis: results of the BAPM/CDSC surveillance study, 1981-84. Arch Dis Child 64:388-394

4. Jason JM 1989 Infectious disease-related deaths of low birth weight infants, United States, 1968 to 1982. Pediatrics 84:296-303

5. Holman RC, Stehr-Green JK, Zelasky MT 1989 Necrotizing enterocolitis mortality in the United States, 1979-1985. Am J Public Health 79:987-991

6. Uauy RD, Fanaroff AA, Korones SB, Phillips E, Phillips J, Wright L 1991 Necrotizing enterocolitis in very low birth weight infants: biodemographic and clinical correlates. J Pediatr 119:630-638

7. Eglow R, Pothoulakis C, Itzkowitz S, Israel E, O'Keane C, Gong D, Gao N, Xu Y, Walker W, LaMont J 1992 Diminished Clostridium difficile toxin A sensitivity in newborn rabbit ileum is associated with decreased toxin $R$ receptor. J Clin Invest 90:822-829

8. Wilde J, Yolken R, Willoughby R, Eiden J 1992 Improved detection of rotavirus shedding by polymerase chain reaction. Lancet 337:323-326

9. Relman DA, Schmidt TM, MacDermott RP, Falkow S 1992 Identification of the uncultured Bacillus of Whipple's disease. N Engl J Med 327:293-298

10. Halac E, Halac J, Begue EF, Casanas J, Indiveri D, Petit J, Figueroa M, Olmas J, Rodriguez L, Oblegon R, Martinez M, Grinblat D, Vilarrodena H 1990 Prenatal and postnatal corticosteroid therapy to prevent neonatal necrotizing enterocolitis: a controlled trial. J Pediatr 117:132-138

11. Eibl MM, Wolf HM, Furnkranz H, Rosenkranz A 1988 Prevention of necrotizing enterocolitis in low-birth-weight infants by IgA-IgG feeding. $\mathrm{N}$ Engl $\mathrm{J}$ Med 319:1-7

12. Lucas A, Cole TJ 1990 Breast milk and neonatal necrotising enterocolitis. Lancet 336:1519-1525

13. Caplan MS, Kelly A, Hsueh W 1992 Endotoxin and hypoxia-induced intestinal necrosis in rats: the role of platelet activating factor. Pediatr Res 31:428-434

14. Gross SJ, Slagle TA 1993 Feeding the low birthweight infant. Clin Perinatol 20:193-209

15. Coombs RC, Morgan MEI, Durbin GM, Booth I, McNeish A 1992 Abnormal gut blood flow velocities in neonates at risk of necrotising enterocolitis. J Pediatr Gastroenterol Nutr 15:13-18

16. Coombs RC, Morgan MEI, Durbin GM, Booth I, McNeish A 1992 Doppler assessment of human neonatal gut blood flow velocities: postnatal adaptation and response to feeds. J Pediatr Gastroenterol Nutr 15:6-12

17. Hackett GA, Campbell S, Gamsu H, Cohen-Overbeek T, Pearch J 1987 Doppler studies in the growth retarded fetus and prediction of neonatal necrotising enterocolitis, haemorrhage, and neonatal morbidity. BMJ 294:13-16

18. Tompkins LS 1992 The use of molecular methods in infectious diseases. N Engl J Med 327:1290-1297 